EUROPEAN JOURNAL OF PURE AND APPLIED MATHEMATICS

Vol. 14, No. 4, 2021, 1457-1466

ISSN 1307-5543 - ejpam.com

Published by New York Business Global

\title{
Fourier Expansion, Integral Representation and Explicit Formula at Rational Arguments of the Tangent Polynomials of Higher-Order
}

\author{
Cristina B. Corcino ${ }^{1,2}$, Roberto B. Corcino ${ }^{1,2, *}$, Jeremar S. Casquejo ${ }^{1}$ \\ 1 Research Institute for Computational Mathematics and Physics, Cebu Normal University, \\ 6000 Cebu City, Philippines \\ 2 Mathematics Department, Cebu Normal University, 6000 Cebu City, Philippines
}

\begin{abstract}
In this paper, Fourier series expansion of Tangent polynomials are derived and the integral representation and explicit formula at rational arguments of these polynomials are established.

2020 Mathematics Subject Classifications: 11B68, 42A16, 11M35

Key Words and Phrases: Genocchi polynomials, Tangent polynomials, Bernoulli polynomials, Euler polynomials, Genocchi polynomials, generating functions, Fourier series, integral representation
\end{abstract}

\section{Introduction}

For $r \in \mathbb{N}$, the higher-order tangent polynomials, $T_{n}^{r}(x)(n \geq 0)$, are defined by the following generating function (see [1])

$$
\left(\frac{2}{e^{2 t}+1}\right)^{r} e^{x t}=\sum_{n=0}^{\infty} T_{n}^{r}(x) \frac{t^{n}}{n !}, \quad|2 t|<\pi .
$$

When $r=1$, the above equation gives the generating function for the classical tangent polynomials (see [2]).

The study of tangent polynomials has become an interesting area for many mathematicians for they possess significant properties that can be found in the field of mathematics and physics (see [3], [4]). Analogues, explicit identities and symmetric properties for tangent polynomials are derived in (see [5], [6], [7]).

In this paper, the researchers derive the Fourier expansion and integral representation of the tangent polynomials of order $r, r \in \mathbb{Z}^{+}$and present an explicit formula of these polynomials at rational arguments using the method of Luo [8].

*Corresponding author.

DOI: https://doi.org/10.29020/nybg.ejpam.v14i4.4152

Email addresses: corcinoc@cnu.edu.ph (C. Corcino), rcorcino@yahoo.com (R. Corcino), casquejoj@cnu.edu.ph (J. Casquejo) 
C. Corcino, R. Corcino, J. Casquejo / Eur. J. Pure Appl. Math, 14 (4) (2021), 1457-1466

\section{Fourier Expansions for Tangent polynomials of Higher-order}

In this section, we give Fourier expansion for tangent polynomials of higher order.

Theorem 2.1. For $0 \leq x \leq 1$,

$$
\begin{aligned}
T_{n}^{r}(x)=2 \cdot n !\left(\frac{2}{\pi}\right)^{r+n} \sum_{k=0}^{\infty} & \sum_{j=0}^{r-1}(-1)^{j}\left(\begin{array}{c}
r+n-j-1 \\
r-j-1
\end{array}\right) \frac{\pi^{j}}{j !} B_{j}^{r}\left(\frac{x}{2}\right) \\
& \times \frac{\cos [(2 k+1) \pi x / 2-(r+n-j) \pi / 2]}{(2 k+1)^{r+n-j}},
\end{aligned}
$$

where $B_{j}^{r}\left(\frac{x}{2}\right)$ denotes the Bernoulli polynomials of order $r$ defined by

$$
\left(\frac{w}{e^{w}-1}\right)^{r} e^{x w}=\sum_{j=0}^{\infty} B_{j}^{r}(x) \frac{w^{n}}{n !} .
$$

Proof. For $r \geq 2$,

$$
\operatorname{Res}\left(f(t), t=t_{k}\right)=\frac{1}{(r-1) !} \lim _{t \rightarrow t_{k}} \frac{d^{r-1}}{d t^{r-1}}\left(t-t_{k}\right)^{r}\left(\frac{2}{e^{2 t}+1}\right)^{r} \frac{e^{x t}}{t^{n+1}} .
$$

Consider the function

$$
\left(t-t_{k}\right)^{r}\left(\frac{2}{e^{2 t}+1}\right)^{r} \frac{e^{x t}}{t^{n+1}}=2^{r} \frac{\left(t-t_{k}\right)^{r}}{\left(e^{2 t}+1\right)^{r}} \frac{e^{x t}}{t^{n+1}}
$$

Writing $\left(e^{2 t}+1\right)^{r}$ as

$$
\begin{aligned}
\left(e^{2 t}+1\right)^{r} & =(-1)^{r}\left(e^{2 t}(-1)-1\right)^{r} \\
& =(-1)^{r}\left(e^{2 t} \cdot e^{-2 t_{k}}-1\right)^{r} \\
& =(-1)^{r}\left(e^{2\left(t-t_{k}\right)}-1\right)^{r},
\end{aligned}
$$

and since $e^{-2 t_{k}}=e^{-2(2 k+1) \frac{\pi}{2} i}=e^{-(2 k+1) \pi i}=-1$, we have

$$
\begin{aligned}
\left(t-t_{k}\right)^{r}\left(\frac{2}{e^{2 t}+1}\right)^{r} \frac{e^{x t}}{t^{n+1}} & =\frac{2^{r}\left(t-t_{k}\right)^{r}}{(-1)^{r}\left(e^{2\left(t-t_{k}\right)}-1\right)^{r}} \cdot \frac{e^{x t}}{t^{n+1}} \\
& =(-1)^{r} \frac{\left(2\left(t-t_{k}\right)\right)^{r}}{\left(e^{2\left(t-t_{k}\right)}-1\right)^{r}} \cdot \frac{e^{x t}}{t^{n+1}} \\
& =(-1)^{r}\left(\sum_{n=0}^{\infty} B_{n}^{r} \frac{\left(2\left(t-t_{k}\right)\right)^{n}}{n !}\right) e^{x t} t^{-(n+1)},
\end{aligned}
$$

where $B_{n}^{r}$ denotes the Bernoulli numbers of order $r$ defined by the generating function 


$$
\left(\frac{w}{e^{w}-1}\right)^{r}=\sum_{n=0}^{\infty} B_{n}^{r} \frac{w^{n}}{n !}
$$

To get the derivative, applying the Leibniz Rule yields

$$
\begin{aligned}
& \frac{d^{r-1}}{d t^{r-1}}\left\{\left(t-t_{k}\right)^{r}\left(\frac{2}{e^{2 t}+1}\right)^{r} \frac{e^{x t}}{t^{n+1}}\right\} \\
& =\frac{d^{r-1}}{d t^{r-1}}\left\{(-1)^{r}\left(\sum_{n=0}^{\infty} B_{n}^{r} \frac{\left(2\left(t-t_{k}\right)\right)^{n}}{n !}\right) e^{x t} t^{-(n+1)}\right\} \\
& =(-1)^{r} \frac{d^{r-1}}{d t^{r-1}}\left\{\left(e^{x t} \sum_{n=0}^{\infty} B_{n}^{r} \frac{\left(2\left(t-t_{k}\right)\right)^{n}}{n !}\right) t^{-(n+1)}\right\} \\
& =(-1)^{r} \sum_{j=0}^{r-1}\left(\begin{array}{c}
r-1 \\
j
\end{array}\right) \frac{d^{r-1-j}}{d t^{r-1-j}} t^{-(n+1)} \cdot \frac{d^{j}}{d t^{j}}\left(e^{x t} \sum_{n=0}^{\infty} B_{n}^{r} \frac{\left(2\left(t-t_{k}\right)\right)^{n}}{n !}\right) \text {, } \\
& \frac{d^{j}}{d t^{j}}\left(e^{x t} \sum_{n=0}^{\infty} B_{n}^{r} \frac{\left.2^{n}\left(t-t_{k}\right)^{n}\right)}{n !}\right) \\
& =\sum_{l=0}^{j}\left(\begin{array}{l}
j \\
l
\end{array}\right) x^{j-l} e^{x t} \sum_{n=l}^{\infty} B_{n}^{r} \frac{2^{n}}{n !}(n)_{l}\left(t-t_{k}\right)^{n-l} \\
& =e^{x t} \sum_{l=0}^{j}\left(\begin{array}{l}
j \\
l
\end{array}\right) x^{j-l} \sum_{n=l}^{\infty} 2^{n} B_{n}^{r} \frac{\left(t-t_{k}\right)^{n-l}}{(n-l) !}, \\
& \frac{d^{r-1}}{d t^{r-1}}\left(\left(t-t_{k}\right)^{r}\left(\frac{2}{e^{2 t}+1}\right)^{r} \frac{e^{x t}}{t^{n+1}}\right) \\
& =(-1)^{r} \sum_{j=0}^{r-1}\left(\begin{array}{c}
r-1 \\
j
\end{array}\right) \frac{d^{r-1-j}}{d t^{r-1-j}} t^{-(n+1)} \\
& \times e^{x t} \sum_{l=0}^{j}\left(\begin{array}{l}
j \\
l
\end{array}\right) x^{j-l} \sum_{n=l}^{\infty} 2^{n} B_{n}^{r} \frac{\left(t-t_{k}\right)^{n-l}}{(n-l) !} .
\end{aligned}
$$

Thus,

$$
\begin{aligned}
\operatorname{Res}\left(f(t), t=t_{k}\right)=\frac{1}{(r-1) !} & \lim _{t \rightarrow t_{k}}(-1)^{r} \sum_{j=0}^{r-1}\left(\begin{array}{c}
r-1 \\
j
\end{array}\right) \frac{d^{r-1-j}}{d t^{r-1-j}} t^{-(n+1)} \\
& \times \lim _{t \rightarrow t_{k}} e^{x t} \sum_{l=0}^{j}\left(\begin{array}{l}
j \\
l
\end{array}\right) x^{j-l} \sum_{n=l}^{\infty} 2^{n} B_{n}^{r} \frac{\left(t-t_{k}\right)^{n-l}}{(n-l) !} .
\end{aligned}
$$


Note that $B_{n}^{r} \frac{\left(t-t_{k}\right)^{n-l}}{(n-l) !} \rightarrow 0$ as $t \rightarrow t_{k}$ except when $n=l$. This gives

$$
\begin{aligned}
& \operatorname{Res}\left(f(t), t=t_{k}\right)= \frac{1}{(r-1) !}(-1)^{r} \sum_{j=0}^{r-1}\left(\begin{array}{c}
r-1 \\
j
\end{array}\right)(-1)^{r-1-j}(n+r-1-j)_{r-1-j} t_{k}^{-(n+r-j)} \\
& \times e^{x t_{k}} \sum_{l=0}^{j}\left(\begin{array}{l}
j \\
l
\end{array}\right) x^{j-l} 2^{l} B_{l}^{r} \\
&= \frac{(-1)^{r}}{(r-1) !} \sum_{j=0}^{r-1} \frac{(r-1) !}{j !(r-1-j) !}(-1)^{r-1-j}(n+r-1-j)_{r-1-j} t_{k}^{-(n+r-j)} \\
& \times e^{x t_{k}} \sum_{l=0}^{j}\left(\begin{array}{l}
j \\
l
\end{array}\right) x^{j-l} 2^{l} B_{l}^{r} \\
&=\sum_{j=0}^{r-1}(-1)^{j-1}\left(\begin{array}{c}
n+r-1-j \\
r-1-j
\end{array}\right) \frac{t_{k}^{j-n-r}}{j !} e^{x t_{k}} 2^{j} \sum_{l=0}^{j}\left(\begin{array}{l}
j \\
l
\end{array}\right) \frac{x^{j-l}}{2^{j-l}} B_{l}^{r} .
\end{aligned}
$$

Recall that $B_{j}^{r}\left(\frac{x}{2}\right)=\sum_{l=0}^{j}\left(\begin{array}{l}j \\ l\end{array}\right) B_{l}^{r}\left(\frac{x}{2}\right)^{j-l}$. Thus,

$$
\begin{aligned}
\operatorname{Res}\left(f(t), t=t_{k}\right) & =\sum_{j=0}^{r-1}(-1)^{j-1} 2^{j}\left(\begin{array}{c}
n+r-1-j \\
r-1-j
\end{array}\right) \frac{t_{k}^{j-n-r}}{j !} e^{x t_{k}} B_{j}^{r}\left(\frac{x}{2}\right) \\
& =\sum_{j=0}^{r-1}(-1)^{j-1} 2^{j}\left(\begin{array}{c}
n+r-1-j \\
r-1-j
\end{array}\right) \frac{B_{j}^{r}\left(\frac{x}{2}\right)}{j !} \frac{e^{x t_{k}}}{t_{k}^{n+r-j}} \\
& =\sum_{j=0}^{r-1}(-1)^{j-1} 2^{j}\left(\begin{array}{c}
r+n-j-1 \\
r-j-1
\end{array}\right) \frac{B_{j}^{r}\left(\frac{x}{2}\right)}{j !} \frac{e^{x t_{k}}}{t_{k}^{r+n-j}} .
\end{aligned}
$$

Taking $t_{k}=\frac{1}{2}(2 k+1) \pi i$, we get

$$
\begin{gathered}
\operatorname{Res}\left(f(t), t=t_{k}\right)=\sum_{j=0}^{r-1}(-1)^{j-1} 2^{j}\left(\begin{array}{c}
r+n-j-1 \\
r-j-1
\end{array}\right) \frac{B_{j}^{r}\left(\frac{x}{2}\right)}{j !} \frac{e^{\frac{1}{2}(2 k+1) \pi i x}}{\left(\frac{1}{2}(2 k+1) \pi i\right)^{r+n-j}} \\
=\frac{1}{\left(\frac{1}{2} \pi i\right)^{r+n}} \sum_{j=0}^{r-1}(-1)^{j-1} 2^{j}\left(\begin{array}{c}
r+n-j-1 \\
r-j-1
\end{array}\right) \frac{\left(\frac{1}{2} \pi i\right)^{j}}{j !} B_{j}^{r}\left(\frac{x}{2}\right) \frac{e^{\frac{1}{2}(2 k+1) \pi i x}}{(2 k+1)^{r+n-j}} .
\end{gathered}
$$

This gives

$$
T_{n}^{r}(x)=n !\left(\frac{2}{\pi i}\right)^{r+n} \sum_{k \in \mathbb{Z}}\left(\sum_{j=0}^{r-1}(-1)^{j}\left(\begin{array}{c}
r+n-j-1 \\
r-j-1
\end{array}\right) \frac{(\pi i)^{j}}{j !} B_{j}^{r}\left(\frac{x}{2}\right)\right) \frac{e^{\frac{1}{2}(2 k+1) \frac{\pi i}{2} x}}{(2 k+1)^{r+n-j}} .
$$


Now, from (3), we look at

$$
i^{-(r+n-j)} \sum_{k \in \mathbb{Z}} \frac{e^{(2 k+1) \frac{\pi i}{2} x}}{(2 k+1)^{r+n-j}} .
$$

Noting that $i^{-(r+n-j)}=e^{-(r+n-j) \pi i / 2}$ and $(-1)^{r+n-j}=e^{(r+n-j) \pi i}$, we see that

$$
\begin{aligned}
i^{-(r+n-j)} \sum_{k \in \mathbb{Z}} \frac{e^{(2 k+1) \frac{\pi i}{2} x}}{(2 k+1)^{r+n-j}} & =i^{-(r+n-j)}\left\{\sum_{k=0}^{\infty} \frac{e^{(2 k+1) \frac{\pi i}{2} x}}{(2 k+1)^{r+n-j}}+(-1)^{r+n-j} \sum_{k=0}^{\infty} \frac{e^{-(2 k+1) \frac{\pi i}{2} x}}{(2 k+1)^{r+n-j}}\right\} \\
& =\sum_{k=0}^{\infty} \frac{e^{[(2 k+1) x / 2-(r+n-j) / 2] \pi i}+e^{-[(2 k+1) x / 2-(r+n-j) / 2] \pi i}}{(2 k+1)^{r+n-j}} \\
& =\sum_{k=0}^{\infty} \frac{2 \cos [(2 k+1) \pi x / 2-(r+n-j) \pi / 2]}{(2 k+1)^{r+n-j}} \\
& =2 \sum_{k=0}^{\infty} \frac{\cos [(2 k+1) \pi x / 2-(r+n-j) \pi / 2]}{(2 k+1)^{r+n-j}}
\end{aligned}
$$

Replacing (4) with (5) in (3), we get the desired formula (2).

\section{Integral representation for Tangent polynomials of Higher-order}

In this section, we establish an integral representation for tangent polynomials of higher order.

Theorem 3.1. For $n \in \mathbb{N}, r \geq 2$, and $0 \leq \mathfrak{R}(x) \leq 1$,

$$
\begin{gathered}
T_{n}^{r}(x)=2^{r+n} \sum_{j=0}^{r-1} \frac{(-1)^{j}}{j !} \cdot \frac{B_{j}^{r}\left(\frac{x}{2}\right)}{(r-j-1) !}\left\{\int_{0}^{\infty} \frac{e^{\pi t} \cos [\pi x / 2-(r+n-j) \pi / 2]}{\cosh (2 \pi t)-\cos (\pi x)} t^{r+n-j-1} d t\right. \\
\left.-\int_{0}^{\infty} \frac{e^{-\pi t} \cos [\pi x / 2+(r+n-j) \pi / 2]}{\cosh (2 \pi t)-\cos (\pi x)} t^{r+n-j-1} d t\right\} .
\end{gathered}
$$

Proof. From (2), we get

$$
\begin{aligned}
T_{n}^{r}(x)=2 \cdot n !\left(\frac{2}{\pi}\right)^{r+n} & \sum_{k=0}^{\infty} \sum_{j=0}^{r-1}(-1)^{j} \frac{(r+n-j-1) !}{(r-j-1) ! n !} \cdot \frac{\pi^{j}}{j !} B_{j}^{r}\left(\frac{x}{2}\right) \\
& \times \frac{\cos [(2 k+1) \pi x / 2-(r+n-j) \pi / 2]}{(2 k+1)^{r+n-j}} \\
= & 2^{r+n+1} \sum_{j=0}^{r-1} \frac{(-1)^{j} B_{j}^{r}\left(\frac{x}{2}\right)}{(r-j-1) ! j !} \cdot \frac{(r+n-j-1) !}{\pi^{r+n-j}} \sum_{k=0}^{\infty} \frac{\cos [(2 k+1) \pi x / 2-(r+n-j) \pi / 2]}{(2 k+1)^{r+n-j}} .
\end{aligned}
$$


We look at

$$
\begin{aligned}
\frac{(r+n-j-1) !}{\pi^{r+n-j}} \sum_{k=0}^{\infty} \frac{\cos [(2 k+1) \pi x / 2-(r+n-j) \pi / 2]}{(2 k+1)^{r+n-j}} & \\
& =\frac{1}{\pi^{r+n-j}} \sum_{k=0}^{\infty} \cos [(2 k+1) \pi x / 2-(r+n-j) \pi / 2] \frac{(r+n-j-1) !}{(2 k+1)^{r+n-j}} .
\end{aligned}
$$

Applying the integral formula

$$
\int_{0}^{\infty} t^{n} e^{-a t} d t=\frac{n !}{a^{n+1}}
$$

for $n \geq 0$ and $\mathfrak{R}(a)>0$, then (8) becomes

$$
\begin{aligned}
& \frac{(r+n-j-1) !}{\pi^{r+n-j}} \sum_{k=0}^{\infty} \frac{\cos [(2 k+1) \pi x / 2-(r+n-j) \pi / 2]}{(2 k+1)^{r+n-j}} \\
& =\frac{1}{\pi^{r+n-j}} \sum_{k=0}^{\infty} \cos [(2 k+1) \pi x / 2-(r+n-j) \pi / 2] \int_{0}^{\infty} t^{r+n-j-1} e^{-(2 k+1) t} d t \\
& =\frac{1}{\pi^{r+n-j}} \int_{0}^{\infty} t^{r+n-j-1} \sum_{k=0}^{\infty} e^{-(2 k+1) t} \cos [(2 k+1) \pi x / 2-(r+n-j) \pi / 2] d t \\
& =\frac{1}{\pi^{r+n-j}} \int_{0}^{\infty} t^{r+n-j-1} \sum_{k=0}^{\infty} e^{-(2 k+1) t}\{\cos [(2 k+1) \pi x / 2] \cos [(r+n-j) \pi / 2] \\
& \quad+\frac{1}{\pi^{r+n-j}} \int_{0}^{\infty}\left\{\cos [(r+n-j) \pi / 2] \sum_{k=0}^{\infty} e^{-(2 k+1) t} \cos [(2 k+1) \pi x / 2]\right. \\
& \left.\quad+\sin [(r+n-j) \pi / 2] \sum_{k=0}^{\infty} e^{-(2 k+1) t} \sin [(2 k+1) \pi x / 2]\right\} t^{r+n-j-1} d t .
\end{aligned}
$$

By making use of

$$
\sum_{k=0}^{\infty} e^{-(2 k+1) t} \sin [(2 k+1) x]=\frac{\sin x \cosh t}{\cosh (2 t)-\cos (2 x)},
$$

and

$$
\sum_{k=0}^{\infty} e^{-(2 k+1) t} \cos [(2 k+1) x]=\frac{\cos x \sinh t}{\cosh (2 t)-\cos (2 x)},
$$

which may be deduced from

$$
\sum_{k=0}^{\infty} e^{(x i-t)(2 k+1)}=\frac{\cos x \sinh t+i \sin x \cosh t}{\cosh (2 t)-\cos (2 x)},
$$


for $t>0$, (9) then becomes

$$
\begin{aligned}
& \frac{(r+n-j-1) !}{\pi^{r+n-j}} \sum_{k=0}^{\infty} \frac{\cos [(2 k+1) \pi x / 2-(r+n-j) \pi / 2]}{(2 k+1)^{r+n-j}} \\
& =\frac{1}{\pi^{r+n-j}} \int_{0}^{\infty}\left\{\cos [(r+n-j) \pi / 2] \frac{\cos \frac{\pi x}{2} \sinh t}{\cosh (2 t)-\cos (\pi x)}\right. \\
& \left.\quad+\sin [(r+n-j) \pi / 2] \frac{\sin \frac{\pi x}{2} \cosh t}{\cosh (2 t)-\cos (\pi x)}\right\} t^{r+n-j-1} d t .
\end{aligned}
$$

Applying the transformation $t=\pi t,(10)$ becomes

$$
\begin{aligned}
\frac{(r+n-j-1) !}{\pi^{r+n-j}} \sum_{k=0}^{\infty} \frac{\cos [(2 k+1) \pi x / 2-(r+n-j) \pi / 2]}{(2 k+1)^{r+n-j}} & \frac{1}{\pi^{r+n-j}} \int_{0}^{\infty}\left\{\cos [(r+n-j) \pi / 2] \frac{\cos \frac{\pi x}{2} \sinh \pi t}{\cosh (2 \pi t)-\cos (\pi x)}\right. \\
& \left.\quad+\sin [(r+n-j) \pi / 2] \frac{\sin \frac{\pi x}{2} \cosh \pi t}{\cosh (2 \pi t)-\cos (\pi x)}\right\} \pi^{r+n-j} t^{r+n-j-1} d t \\
= & \int_{0}^{\infty}\left\{\frac{\cos [(r+n-j) \pi / 2] \cos \frac{\pi x}{2}\left(e^{\pi s}-e^{-\pi s}\right)}{2[\cosh (2 \pi t)-\cos (\pi x)]}\right. \\
& \left.+\frac{\sin [(r+n-j) \pi / 2] \sin \frac{\pi x}{2}\left(e^{\pi s}+e^{-\pi s}\right)}{2[\cosh (2 \pi t)-\cos (\pi x)]}\right\} t^{r+n-j-1} d t \\
= & \frac{1}{2} \int_{0}^{\infty}\left\{\frac{e^{\pi s}\left(\cos [(r+n-j) \pi / 2] \cos \frac{\pi x}{2}+\sin [(r+n-j) \pi / 2] \sin \frac{\pi x}{2}\right)}{\cosh (2 \pi t)-\cos (\pi x)}\right. \\
& \left.\quad-\frac{e^{-\pi s}\left(\cos [(r+n-j) \pi / 2] \cos \frac{\pi x}{2}-\sin [(r+n-j) \pi / 2] \sin \frac{\pi x}{2}\right)}{\cosh (2 \pi t)-\cos (\pi x)}\right\} t^{r+n-j-1} d t \\
= & \frac{1}{2} \int_{0}^{\infty} \frac{e^{\pi s} \cos [\pi x / 2-(r+n-j) \pi / 2]-e^{-\pi s} \cos [\pi x / 2+(r+n-j) \pi / 2]}{\cosh (2 \pi t)-\cos (\pi x)} t^{r+n-j-1} d t .
\end{aligned}
$$

Applying (11) to (7), we get the desired formula (6).

\section{Explicit formula for Tangent polynomials of Higher-order at rational arguments}

In this section, we obtain an explicit formula for tangent polynomials of higher order at rational arguments by applying the Fourier expansion (2). Here let $\mathbb{Z}_{0}^{-}=$ $\{0,-1,-2, \cdots\}$ denote the set of nonpositive integers.

Theorem 4.1. For $n, q \in \mathbb{N}$ and $p \in \mathbb{Z}$,

$$
T_{n}^{r}\left(\frac{2 p}{q}\right)=\frac{2 \cdot n !}{(q \pi)^{r+n}} \sum_{j=0}^{r-1}(-1)^{j}\left(\begin{array}{c}
r+n-j-1 \\
r-j-1
\end{array}\right) \frac{(2 q \pi)^{j}}{j !} B_{j}^{r}\left(\frac{p}{q}\right)
$$


C. Corcino, R. Corcino, J. Casquejo / Eur. J. Pure Appl. Math, 14 (4) (2021), 1457-1466

$$
\times \sum_{l=1}^{q} \zeta\left(r+n-j, \frac{2 l-1}{2 q}\right) \cos \left[\frac{(2 l-1) p \pi}{q}-\frac{(r+n-j) \pi}{2}\right]
$$

where

$$
\zeta(s, a)=\sum_{n=0}^{\infty} \frac{1}{(n+a)^{s}},
$$

for $\mathfrak{R}(s)>1$ and $a \notin \mathbb{Z}_{0}^{-}$is Hurwitz zeta function.

Proof. We look at

$$
\sum_{k=0}^{\infty} \frac{\cos [(2 k+1) \pi x / 2-(r+n-j) \pi / 2]}{(2 k+1)^{r+n-j}} .
$$

Replacing $k$ with $k-1$ :

$$
\sum_{k=0}^{\infty} \frac{\cos [(2 k+1) \pi x / 2-(r+n-j) \pi / 2]}{(2 k+1)^{r+n-j}}=\sum_{k=1}^{\infty} \frac{\cos [(r+n-j) \pi / 2-(2 k-1) \pi x / 2]}{(2 k-1)^{r+n-j}}
$$

Applying the elementary series identity

$$
\sum_{k=1}^{\infty} f(k)=\sum_{l=1}^{q} \sum_{k=0}^{\infty} f(q k+l), \quad q \in \mathbb{N},
$$

(15) becomes

$$
\begin{aligned}
\sum_{k=0}^{\infty} & \frac{\cos [(2 k+1) \pi x / 2-(r+n-j) \pi / 2]}{(2 k+1)^{r+n-j}} \\
& =\sum_{l=1}^{q} \sum_{k=0}^{\infty} \frac{\cos [(r+n-j) \pi / 2-(2 q k+2 l-1) \pi x / 2]}{(2 q k+2 l-1)^{r+n-j}} \\
& =\sum_{l=1}^{q} \sum_{k=0}^{\infty} \frac{\cos [(r+n-j) \pi / 2-(2 l-1) \pi x / 2-q k \pi x]}{\left[2 q\left(k+\frac{2 l-1}{2 q}\right)\right]^{r+n-j}} \\
& =\sum_{l=1}^{q} \sum_{k=0}^{\infty} \frac{\cos [(r+n-j) \pi / 2-(2 l-1) \pi x / 2-q k \pi x]}{(2 q)^{r+n-j}} \cdot \frac{1}{\left(k+\frac{2 l-1}{2 q}\right)^{r+n-j}} .
\end{aligned}
$$

Setting $x=2 p / q,(16)$ becomes

$$
\begin{aligned}
& \sum_{k=0}^{\infty} \frac{\cos [(2 k+1) \pi x / 2-(r+n-j) \pi / 2]}{(2 k+1)^{r+n-j}} \\
& \quad=\sum_{l=1}^{q} \sum_{k=0}^{\infty} \frac{\cos [(r+n-j) \pi / 2-(2 l-1) p \pi / q-2 \pi(p k)]}{(2 q)^{r+n-j}} \cdot \frac{1}{\left(k+\frac{2 l-1}{2 q}\right)^{r+n-j}}
\end{aligned}
$$




$$
\begin{aligned}
& =\frac{1}{(2 q)^{r+n-j}} \sum_{l=1}^{q} \sum_{k=0}^{\infty} \cos [(r+n-j) \pi / 2-(2 l-1) p \pi / q] \cdot \frac{1}{\left(k+\frac{2 l-1}{2 q}\right)^{r+n-j}} \\
& =\frac{1}{(2 q)^{r+n-j}} \sum_{l=1}^{q} \cos [(r+n-j) \pi / 2-(2 l-1) p \pi / q] \sum_{k=0}^{\infty} \frac{1}{\left(k+\frac{2 l-1}{2 q}\right)^{r+n-j}} .
\end{aligned}
$$

By (13), (17) becomes

$$
\begin{aligned}
& \sum_{k=0}^{\infty} \frac{\cos [(2 k+1) \pi x / 2-(r+n-j) \pi / 2]}{(2 k+1)^{r+n-j}} \\
& \quad=\frac{1}{(2 q)^{r+n-j}} \sum_{l=1}^{q} \cos \left[\frac{(2 l-1) p \pi}{q}-\frac{(r+n-j) \pi}{2}\right] \zeta\left(r+n-j, \frac{2 l-1}{2 q}\right) .
\end{aligned}
$$

Replacing (14) with (18) in (2), we obtain the desired formula (12).

\section{Acknowledgements}

The authors would like to thank the anonymous referees for reviewing the paper thoroughly The authors would also like to thank Cebu Normal University (CNU) for funding this research project through its Research Institute for Computational Mathematics and Physics (RICMP).

\section{References}

[1] Ryoo, C. S., Multiple tangent zeta function and tangent polynomials of higher order, Adv. Studies Theor. Phys 8(10) (2014), 457-462.

[2] Ryoo, C. S., A note on the tangent numbers and polynomials, Adv. Studies Theor. Phys 7(9) (2013), 447-454.

[3] Ryoo, C. S., A numerical investigation on the zeros of the tangent polynomials, $J$. Appl. Math. Info. 32(3-4) (2014), 315-322.

[4] Ryoo, C. S., On the twisted q-Tangent numbers and polynomials, Appll. Math. Sci. $\mathbf{7}(99)$ (2013), 4935-4941.

[5] Ryoo, C. S., Explicit Identities for the Generalized Tangent Polynomials, Nonlinear Analysis and Differential Equations 6(1) (2018), 43 - 51.

[6] Ryoo, C. S., On the analogues of Tangent numbers and polynomials associated with p-adic integral on $Z_{p}$, Appl. Math. Sci. 7(64) (2013), 3177-3183.

[7] Ryoo, C. S., A note on the symmetric properties for the tangent polynomials, Int. J. Math. Anal. 7(52) (2013), 2575-2581. 
[8] Luo, Q-M., Fourier expansions and integral representations for the Genocchi polynomials, J. Integer Seq. 12(1) (2009), Article 09.1.4. 